\title{
Phylogenetic relationships of twenty Gymnothorax species based on cytochrome $b$ sequence data
}

\author{
M. Du' ${ }^{1}$ S.W. Yin ${ }^{2}$ and B.Z. Niu ${ }^{1}$ \\ ${ }^{1}$ Key Lab for Quality, Efficient cultivation and Security Control of Crops in \\ Colleges and University of Yunnan Province, College of Life Sciences and \\ Technology, Honghe University, Mengzi, Yunnan Province, China \\ ${ }^{2}$ College of Life Sciences, Nanjing Normal University, Nanjing, China \\ Corresponding author: S.W. Yin \\ E-mail: yinshaowu@163.com
}

Genet. Mol. Res. 15 (2): gmr. 15028157

Received November 26, 2015

Accepted January 18, 2016

Published May 20, 2016

DOI http://dx.doi.org/10.4238/gmr.15028157

\begin{abstract}
To study the phylogenetic relationships of the genus Gymnothorax (moray eels) distributed in South China Sea, polymerase chain reactions were performed, and the amplification products were sequenced by cloning into the PMD18T-vector (TaKaRa). The entire gene sequences encoding cytochrome $b(1140 \mathrm{bp})$ for 16 Gymnothorax (G. flavimarginatus, G. meleagris, G. undulates, G. reticularis, G. reevesi, G. melanospilus, G. rueppeliae, G. javanicus, G. chilospilus, G. pseudothyrsoideus, G. fimbriatus, G. hepaticus, G. berndti, G. curostus, G. favagineus, and G. margaritophorus) were obtained. Four additional Gymnothorax sequences from GenBank were also included. The nucleotide composition, genetic distances, and base substitution saturation analysis were calculated using the MEGA 5.0 Software. Phylogenetic analysis was performed using maximum-parsimony, maximum-likelihood (ML), and neighbor-joining (NJ). The results were as follows: 1) base-substitution saturation analysis suggested that both in third codon positions, and the full-length cytochrome $b$ data
\end{abstract}


set, Ts are not saturated, but Tv substitutions may be saturated, 2) the genus Gymnothorax, native to the South China Sea, is divided into four distinct clades, with two clades in the NJ and ML trees, and 3) according to our experimental data, G. melanospilus (Bleeker, 1855) and G. favagineus (Bloch and Schneider, 1801) are the same species.

Key words: Gymnothorax; Mitochondrial DNA; Cytochrome $b$; Phylogenetic relationship

\section{INTRODUCTION}

Moray eels (Gymnothorax), belonging to the family Muraenidae, are widely distributed in the world's tropical and semi-tropical oceans. The family Muraenidae is one of the most abundant and widespread of all eel families. It is estimated to contain 200 species or more belonging to 15 genera. The genus Gymnothorax (moray eels) contains approximately 120 species worldwide (Tesch, 1977; Castle, 1994; Paxton and Eschmeyer, 1994; Kuiter, 1996). Gymnothorax have no pectoral fins or pelvic fins and are found in all tropical and temperate oceans around the world. Their streamlined bodies are adapted for hunting within the crevices of the reef (LoweMcConnell, 1987; Bohlke and Chaplin, 1993; Randall, 1996). In recent years, Gymnothorax have become the subject of many investigations. For example, Gilbert et al. (2005) estimated the density and biomass of moray eels (Muraenidae). In another study, Ling reported a decrease in the $\mathrm{Sr} / \mathrm{Ca}$ ratios in the otoliths of Gymnothorax reticularis during metamorphosis (Ling et al., 2005). In addition, Tamburrini et al. (2001) studied the structure/function relationships of the brown moray (Gymnothorax unicolor) hemoglobin system. However, there is still debate on the number of Gymnothorax species that inhabit the South and East China Seas. For example, Cheng and Zheng (1987) proposed that there are 20 Gymnothorax species in both South and East China Seas. However, Shen (1993) maintains that there are 27 Gymnothorax species. Furthermore, major criteria for the identification of Gymnothorax, which include ratios of morphological measurements, coloration pattern, variegated markings, and dentition, are vague because most Gymnothorax species are similar in morphology. In addition, their phylogenetic relationships have not been well established. Further studies are needed to resolve these problems.

Throughout the last decade, molecular phylogenetic techniques have led to important changes in the way we understand the evolution of marine life. The number of species for which appropriate sequence information is available has been increasing rapidly, and the methods used have become more sophisticated (Avise, 1994; Stepien and Kocher, 1997). Mitochondrial DNA (mt DNA) can be a powerful molecular marker for reconstructing evolutionary lineages for animals (Avise, 1994). The gene encoding cytochrome $b$ is the most widely used gene for phylogenetic research, and has been the most prevalent source of sequence data. In recent years, the nucleotide sequence of the cytochrome $b$ gene has been widely used in fish taxonomy studies (Song et al., 1998; Thomas et al., 2000; Harris et al., 2002; Bernardi et al., 2003; Wu et al., 2014).

In this paper, we describe a fresh attempt to assess the phylogenetic relationships among different species of the genus Gymnothorax via sequence analysis of mtDNA cytochrome $b$ genes sequences. Our objectives were 1) to clarify the taxonomic status of different moray eels in the genus Gymnothorax of the family Muraenidae and to 2) investigate the status of the moray eels' idioplasm resources for the South China Sea. 


\section{MATERIAL AND METHODS}

\section{Sample collection}

A total of 23 species ( 20 in-group and 3 out-group) were used for the study to evaluate phylogenetic relationships within the genus Gymnothorax. The cytochrome $b$ genes of four species were obtained from GenBank. Other specimens were collected from fish markets in Haikou and Sanya, and from fishing vessels that fished near Xisha and Nansha Island in the South China Sea. Species were initially identified morphologically and identification was confirmed upon being deposited in the lab. Two or three individuals from each species were used for the sequence analysis. The specimens were frozen immediately after being dissected and later kept at $-80^{\circ} \mathrm{C}$.

\section{DNA extraction, amplification, and sequencing}

The crude DNA samples used in the study were prepared from the muscle or tail fin tissue (Kocher et al., 1989) of 16 Gymnothorax species (Table 1). About $0.1 \mathrm{~g}$ tissue was homogenized in $1 \mathrm{~mL}$ digestion buffer $(10 \mathrm{mM}$ Tris-HCl, $\mathrm{pH}$ 8.0, 2 mM EDTA, $10 \mathrm{mM} \mathrm{NaCl}$, $1 \%$ SDS, $10 \mathrm{mg} / \mathrm{mL}$ dithiothreitol, $0.5 \mathrm{mg} / \mathrm{mL}$ Proteinase $\mathrm{K}$ ) and incubated at $37^{\circ} \mathrm{C}$ for 8 to $16 \mathrm{~h}$, followed by a standard phenol and chloroform extraction. The DNA samples were preserved in $50 \mu \mathrm{L}$ TE buffer.

Table 1. Sources of sequence data and samples.

\begin{tabular}{|c|c|c|}
\hline Species & Location & Cytochrome $b$ GenBank accession No. \\
\hline G. kidako & Japan & AP002976 \\
\hline G. flavimarginatus & The South China Sea & EU085360 \\
\hline G. meleagris & The South China Sea & EU085361 \\
\hline G. undulates & The South China Sea & EU085362 \\
\hline G. reticularis & The South China Sea & EU085363 \\
\hline G. reevesi & The South China Sea & EU085364 \\
\hline G. melanospilus & The South China Sea & EU085365 \\
\hline G. rueppeliae & The South China Sea & EU085366 \\
\hline G. javanicus & The South China Sea & EU085367 \\
\hline G. chilospilus & The South China Sea & EU085368 \\
\hline G.pseudothyrsoideus & The South China Sea & EU085369 \\
\hline G. fimbriatus & The South China Sea & EU085370 \\
\hline G. hepaticus & The South China Sea & EU085371 \\
\hline G. berndti & The South China Sea & EU085372 \\
\hline G. curostus & The South China Sea & EU085373 \\
\hline G. favagineus & The South China Sea & EU085374 \\
\hline G. margaritophorus & The South China Sea & EU170376 \\
\hline G.polygonius & Spain & DQ197954 \\
\hline G. unicolor & Spain & DQ197955 \\
\hline G. maderensis & Spain & DQ197953 \\
\hline
\end{tabular}

Sequences for G. kidako, G. polygonius, G. unicolor, and G. maderensis were obtained from GenBank.

A total of 4 PCR primers for cytochrome $b$ genes were designed using the Primer Premier 5.0 Software based on the Gymnothorax kidako mitochondrial genome sequence (Inoue et al., 2003). Primer pairs GYM-Cytb-sen1, GYM-Cytb-ant1, GYM-Cytb-sen2, and GYM-Cytbant2 were used to amplify the full-length sequences of the genes encoding cytochrome $b$. The sequences of those primers are as follows: 5'-CCAGGACCAATGATACGAAA-3' (GYM- 
Cytb-sen1); 5'-AAATTAAGCTACTAGGGCGC-3' (GYM-Cytb-ant1); 5'-CCTTTAATGGCA AACCTACG-3' (GYM-Cytb-sen2); 5'-TAGGGCGCTATCAGTCAAGT-3' (GYM-Cytbant2). PCR amplifications were performed using Nested PCR: the first PCR was performed using the total DNA as the template and the second PCR was performed using the products of the first PCR as the template.

PCR amplifications for cytochrome $b$ were performed in a mixture containing, in a final volume of $25 \mu \mathrm{L}: 16.2 \mu \mathrm{L} \mathrm{ddH}_{2} \mathrm{O}, 1 \mu \mathrm{L}$ crude DNA as the template, $2.5 \mu \mathrm{L}$ dNTP, 2.5 $\mu \mathrm{L}$ Ex Taq buffer containing $\mathrm{MgCl}_{2}, 1.3 \mu \mathrm{L}$ of each primer, $0.2 \mu \mathrm{L}$ Ex Taq DNA polymerase (TaKaRa Biotechnology Co., Ltd, Dalian, China). All amplifications were performed using the following PCR profile: a preliminary denaturation at $94^{\circ} \mathrm{C}$ for 4 min followed by 35 cycles consisting of $94^{\circ} \mathrm{C}$ for $30 \mathrm{~s}, 50^{\circ} \mathrm{C}$ for $30 \mathrm{~s}, 72^{\circ} \mathrm{C}$ for $2 \mathrm{~min}$, and a final extension at $72^{\circ} \mathrm{C}$ for $8 \mathrm{~min}$. The amplification products were separated by electrophoresis through a $1.5 \%$ agarose gel, and the correct-size bands were cut out and purified using the Sangon Gel Extraction Kit, and then cloned into the PMD18T-vector (TaKaRa) and sequenced using a 3770 DNA Sequencer (ABI PRISM) according to the manufacturer instructions. These sequences for the gene encoding cytochrome $b$ for the 16 Gymnothorax species were submitted to GenBank, and the series of accession numbers are listed in Table 1.

\section{Sequence alignment and phylogenetic analyses}

The cytochrome $b$ gene sequences were aligned manually as they are the same length (1140 $\mathrm{bp})$. The aligned DNA sequences were analyzed using the statistical analysis system in the MEGA program (Tamura et al., 2011). To calculate the nucleotide base composition bias and the $\mathrm{Ts} / \mathrm{Tv}$ ratio, base substitution saturation analysis was performed on the gene sequence encoding cytochrome $b$.

Phylogenetic trees were constructed using three methods of the maximum- parsimony (MP), maximum-likelihood (ML), and neighbor-joining (NJ) analyses implemented in PAUP*, version 4.0b10 (Swofford, 2002). Maximum parsimony tree searches were performed in PAUP* using the heuristic search option, with 100 random-addition sequences, tree-bisectionreconnection branch swapping, and collapsing zero-length branches. The reliability of nodes was assessed using 1000 bootstrap replicates (Felsenstein, 1985), and in each case the heuristic search was limited to a maximum of 10,000 saved trees. Consistency and retention indices (CI and RI, respectively) were generated using the computer program PAUP* $4.0 \mathrm{~b} 10$ for the parsimony tree. Skewness values (Hillis and Huelsenbeck, 1992) were estimated from random samples of 10,000 trees generated by PAUP* 4.0b10. Anguilla marmorata, A. japonica, and Ophisurus macrorhynchos, which were obtained from GenBank, were used as an out group for the analysis.

The maximum-likelihood analyses (heuristic search with random addition and ten repetitions, TBR branch swapping, starting branch length using Rogers-Swofford method, and multi-trees on; Rogers and Swofford, 1999) were run using the GTR (general-time-reversible model; Goldman and Yang, 1994). The neighbor-joining (NJ) trees were generated using Kimura 2-parameter (Kimura, 1980) model by PAUP* 4.0b10.

\section{RESULTS}

\section{Sequence data}

All the gene sequences encoding cytochrome $b$ for the 16 Gymnothorax species 
aligned well, and were deposited in GenBank (Table 1). In this study, 20 cytochrome $b$ encoding sequences were analyzed (4 cytochrome $b$ sequences were obtained from GenBank; the accession numbers are listed in Table 1). Mean base composition showed an anti-G bias (Table 2 and Figure 1), which is characteristic for this mitochondrial gene (Cantatore et al., 1994; Briolay et al., 1998). The low G content (mean: 15.4\%) and the almost identical A, C, and $\mathrm{T}$ contents (mean: 25.7, 28.7, and $30.2 \%$, respectively) were similar to those in previous studies on Cyprinidae and Cichlids fish cytochrome $b$ sequences (Briolay et al., 1998; Martin and Bermingham, 1998; Perdices et al., 2004). The A and T content (55.9\%) was higher than that of $\mathrm{G}$ and $\mathrm{C}(44.1 \%)$. The total $\mathrm{A}, \mathrm{T}, \mathrm{C}$, and $\mathrm{G}$ content in the first codon showed little difference, and no nucleotide compositional bias was found. The $\mathrm{T}$ content in the second codon was much higher than that of the other three bases (41.1\%); but the $\mathrm{G}$ content in the third codon was much lower than that of $\mathrm{A}, \mathrm{T}$, and $\mathrm{C}(8.8 \%)$ (Figure 1). This pattern was commonly observed in Zebrafish (Xiang et al., 2004).

Table 2. Nucleotide composition comparison for the 20 species of Gymnothorax studied.

\begin{tabular}{|c|c|c|c|c|c|c|c|c|c|c|}
\hline \multirow[t]{2}{*}{ Species } & \multicolumn{4}{|c|}{ Percentage (\%) } & \multirow[t]{2}{*}{ Total } & \multicolumn{4}{|c|}{ Percentage (\%) } & \multirow[t]{2}{*}{ Pos\#1 } \\
\hline & $\mathrm{A}$ & $\mathrm{T}$ & $\mathrm{C}$ & $\mathrm{G}$ & & A-1 & $\mathrm{T}-1$ & $\mathrm{C}-1$ & G-1 & \\
\hline G. kidako & 25.7 & 30.9 & 28.6 & 14.8 & 1140 & 24.5 & 24.5 & 26.3 & 24.7 & 380 \\
\hline G. fimbriatus & 25.6 & 31.1 & 27.9 & 15.4 & 1140 & 24.5 & 24.7 & 25.8 & 25.0 & 380 \\
\hline G. meleagris & 27.1 & 27.9 & 30.7 & 14.3 & 1140 & 24.5 & 22.6 & 27.6 & 25.3 & 380 \\
\hline G. undulates & 26.0 & 29.6 & 29.4 & 15.1 & 1140 & 24.5 & 25.3 & 25.0 & 25.3 & 380 \\
\hline G. reticularis & 25.5 & 31.1 & 27.8 & 15.5 & 1140 & 24.5 & 24.7 & 25.8 & 25.0 & 380 \\
\hline G. pseudothyrsoideus & 25.4 & 31.4 & 27.6 & 15.5 & 1140 & 24.7 & 24.7 & 25.8 & 24.7 & 380 \\
\hline G. chilospilus & 25.7 & 31.1 & 27.8 & 15.4 & 1140 & 24.5 & 24.7 & 25.8 & 25.0 & 380 \\
\hline G. reevesi & 25.4 & 30.9 & 28.1 & 15.6 & 1140 & 24.2 & 24.5 & 25.8 & 25.5 & 380 \\
\hline G. melanospilus & 25.5 & 29.1 & 29.5 & 15.9 & 1140 & 25.5 & 25.5 & 24.7 & 24.2 & 380 \\
\hline G. flavimarginatus & 25.4 & 31.1 & 27.8 & 15.6 & 1140 & 24.5 & 24.7 & 25.8 & 25.0 & 380 \\
\hline G. rueppeliae & 25.6 & 31.1 & 27.9 & 15.4 & 1140 & 24.7 & 24.7 & 25.8 & 24.7 & 380 \\
\hline G. margaritophorus & 25.7 & 29.6 & 29.6 & 15.2 & 1140 & 24.5 & 25.3 & 25.0 & 25.3 & 380 \\
\hline G. javanicus & 25.5 & 31.1 & 27.8 & 15.5 & 1140 & 24.5 & 24.5 & 26.1 & 25.0 & 380 \\
\hline G. hepaticus & 26.4 & 29.3 & 29.1 & 15.2 & 1140 & 24.7 & 24.7 & 25.5 & 25.0 & 380 \\
\hline G. berndti & 25.5 & 29.5 & 29.7 & 15.3 & 1140 & 24.5 & 25.3 & 25.0 & 25.3 & 380 \\
\hline G. curostus & 25.7 & 31.0 & 27.9 & 15.4 & 1140 & 25.0 & 25.8 & 24.7 & 24.5 & 380 \\
\hline G. favagineus & 25.5 & 29.2 & 29.5 & 15.8 & 1140 & 25.0 & 25.8 & 24.7 & 24.5 & 380 \\
\hline G. polygonius & 31.2 & 28.1 & 26.1 & 14.6 & 1140 & 24.2 & 26.3 & 24.2 & 25.3 & 380 \\
\hline G. maderensis & 29.7 & 28.1 & 26.4 & 15.8 & 1140 & 26.1 & 24.2 & 23.9 & 25.8 & 380 \\
\hline G. unicolor & 28.8 & 30.4 & 24.4 & 16.4 & 1140 & 24.5 & 25.8 & 23.9 & 25.8 & 380 \\
\hline Overall & 30.2 & 28.7 & 25.7 & 15.4 & 1140 & 24.8 & 25.6 & 24.5 & 25.1 & 380 \\
\hline
\end{tabular}

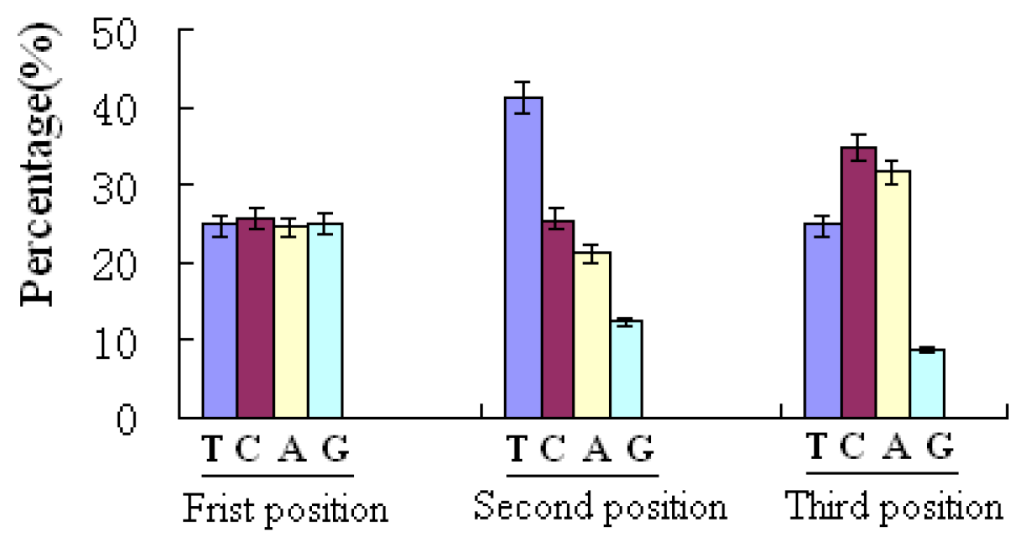

Figure 1. Mean nucleotide base composition of 20 Gymnothorax species (moray eels) at the three codon positions. 
Among these 20 cytochrome $b$ encoding gene sequences, 433 (37.9\%) variables were observed, and 354 are parsimony-informative sites. More than $83.7 \%$ of all informative sites are third codon position substitutions. This observation was consistent with the high evolution rate of the third codon position. This pattern of nucleotide substitutions in Gymnothorax species is very similar to that observed for sharks (Martin, 1995). The transition (Ts)/transversion (Tv) rate ratios were $k_{1}=17.427$ (purines) and $k_{2}=9.951$ (pyrimidines). The rate of Ts substitutions was much higher than the rate of Tv substitutions, and the overall Ts/Tv bias was $2.3(R)$ (Tamura et al., 2011).

To assess the pattern of variation at the third codon positions and the full-length cytochrome $b$ data set, the number of Ts and Tv were plotted against the uncorrected p-distance, for all pairwise comparisons. This analysis showed that in both the third codon positions and the full-length cytochrome $b$ data set, Ts are not saturated and instead show a straight-line trend, but Tv substitutions may be saturated, because the Tv trend plateaued when the uncorrected p-distance reached 0.25 in the graph (Figure 2).
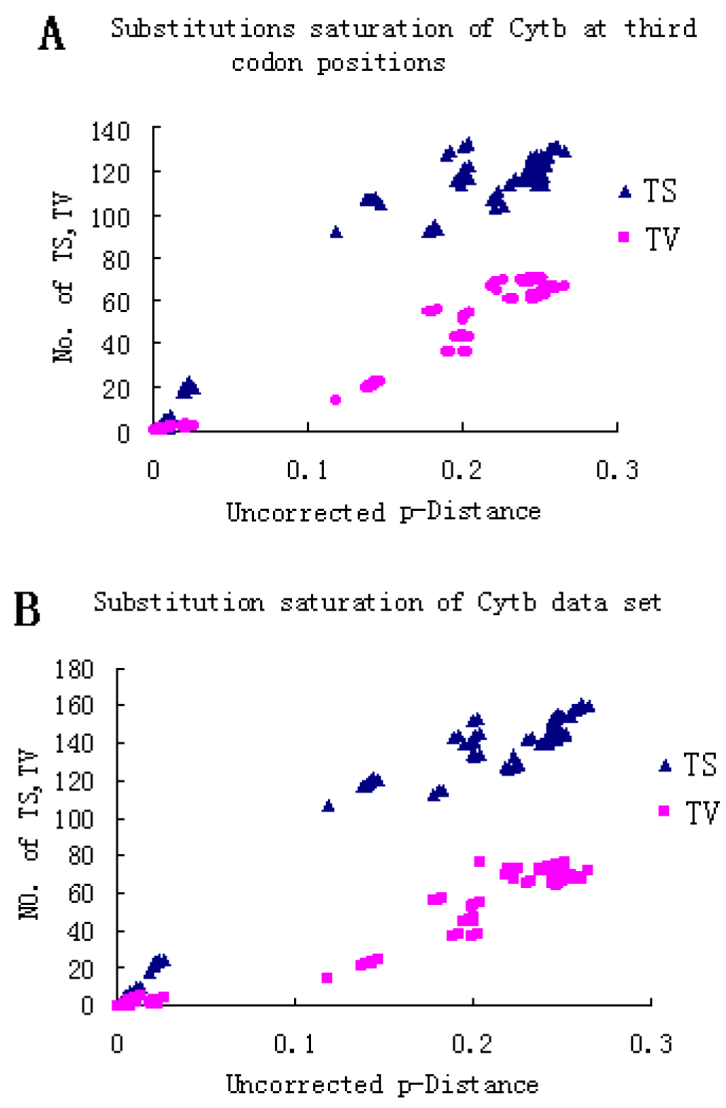

Figure 2. Substitution pattern at the third codon positions (A) and full length sequence (B) of the gene encoding cytochrome $b$. The number of transition (Ts) and transversion (Tv) substitutions is plotted against the uncorrected p-distance considering all sites. Each point represents a pairwise comparison among species. Both in the third codon positions, and the full-length cytochrome $b$ data set, Ts are not saturated, but Tv substitutions may be saturated. The comparisons did not include outgroup taxa. 
A summary of the genetic distances among G. kidako (from Japan), 3 Gymnothorax species (from Spain), and 16 species inhabiting the South China Sea is shown in Table 3. The average difference in base composition for the 16 species from the South China Sea, and $G$. Kidako (from Japan), is $15.0 \%$, which is lower than that of the 3 species of Gymnothorax (22.4\%) from Spain. This result suggests that the evolutionary rate of the Gymnothorax in the western Pacific, including Japan and the Southeast China Sea, is lower than that of the Gymnothorax (22.4\%) from Spain. However, it is strange that the pairwise distance data analysis revealed only small genetic differences among G. fimbriatus, G. reticularis, G. chilospilus, G. rueppeliae, G. javanicus, G. reevesi, and G. flavimarginatus (0.4-0.8\%, Table $3)$. These species exhibit great morphological differences (coloration patterns or variegated markings), which further confirms that the evolutionary rate of Gymnothorax from the western Pacific is lower.

\footnotetext{
Table 3. Summary of the mean pairwise distance (\%) of sequences encoding cytochrome $b$ among the 20 species of Gymnothorax analyzed.

\begin{tabular}{|c|c|c|c|c|c|c|c|c|c|c|c|c|c|c|c|c|c|c|c|c|}
\hline & 1 & 2 & 3 & 4 & 5 & 6 & 7 & 8 & 99 & 10 & 11 & 12 & 13 & 14 & 15 & 16 & 17 & 18 & 19 & \\
\hline$\frac{1}{2}$ & 14.2 & & & & & & & & & & & & & & & & & & & \\
\hline & 13.8 & 0.40 & & & & & & & & & & & & & & & & & & \\
\hline & 20.0 & 22.7 & 22.4 & & & & & & & & & & & & & & & & & \\
\hline & $\begin{array}{r}20.3 \\
13.3\end{array}$ & $\frac{22.5}{0.5}$ & 22. & $\frac{16.9}{22}$ & & & & & & & & & & & & & & & & \\
\hline 7 & $\frac{10.5}{13.5}$ & $\frac{1.40}{220}$ & 2.10 & $\frac{2.24}{220}$ & $\frac{120}{222}$ & 190 & & & & & & & & & & & & & & \\
\hline 8 & 14.1 & $\frac{1}{0.70}$ & 0.0 & 2.2. & $\frac{22.3}{22.3}$ & 0 & 2.20 & & & & & & & & & & & & & \\
\hline & 13.8 & 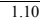 & 0.81 & 22.1 & 21.8 & 0.70 & 2.20 & 1.10 & & & & & & & & & & & & \\
\hline 10 & 21.5 & & 22. & 18.6 & 19.6 & & 22.5 & 22.7 & 22.5 & & & & & & & & & & & \\
\hline & 14.2 & 0.7 & 0.4 & 22.6 & 22.4 & 0.40 & 2.20 & 0.70 & 1.10 & 23.0 & & & & & & & & & & \\
\hline & 20.1 & 22.2 & 21.8 & 16.7 & 1.80 & 21.7 & 22.0 & 22.1 & 21.8 & 18.9 & 22.1 & & & & & & & & & \\
\hline 14 & 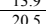 & .50 & 0.36 & 2.25 & 22.2 & 0.20 & 2.10 & 0.50 & 0.90 & 22.8 & 0.40 & 22.0 & & & & & & & & \\
\hline & 20.6 & 22 & 22 & 17 & 21 & 20 & 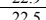 & 225 & 222 & 102 & 225 & 04 & 223 & 192 & & & & & & \\
\hline 16 & 14.3 & 1.00 & 0.7 & 23.0 & 22.7 & 0.60 & 2.50 & 1.00 & 1.30 & 23.3 & 0.80 & 22.4 & 0.60 & 24.3 & 22.8 & & & & & \\
\hline & 3 & & 22. & 18.3 & & & & & 22.6 & 0.50 & 23. & & & & & 23.3 & & & & \\
\hline$\frac{18}{18}$ & 23.9 & 2.0 & 23. & 21.2 & 2.3 & 23. & 23.2 & 23.6 & 23.8 & 22.9 & 23.8 & 23.2 & 23.7 & 20.9 & 23.5 & 24.2 & 22.6 & & & \\
\hline$\frac{19}{20}$ & 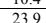 & $\frac{1}{24}$ & 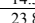 & $\frac{199 .}{22.4}$ & $\frac{21.8}{22.3}$ & $\frac{1}{23}$ & $\frac{1}{23}$ & 240 & 240 & $\frac{2224}{224}$ & $\frac{1}{24 .}$ & $\frac{122.23}{232}$ & 1239 & 228 & 236 & $\frac{1}{244}$ & 222 & 20.8 & 239 & \\
\hline
\end{tabular}

$1=$ G. kidako; $2=$ G. fimbriatus; $3=$ G. flavimarginatus $; 4=$ G. meleagris; $5=$ G. undulates $; 6=$ G. reticularis; $7=$ G. pseudothyrsoideus $; 8=$ G. chilospilus $; 9=$ G. reevesi $; 10=$ G. melanospilus $; 11=$ G. rueppeliae $; 12=G$. margaritophorus $; 13=$ G. javanicus $; 14=$ G. hepaticus $; 15=$ G. berndti $; 16=$ G. curostus $; 17=$ G. favagineus $; 18$ $=$ G. unicolor $; 19=$ G. polygonius; $20=$ G. maderensis.
}

\section{Molecular phylogeny}

The left-skew $(\mathrm{g} 1=-0.54, p=0.01)$ for the random trees indicates that the cytochrome $b$ data matrix contains a phylogenetic signal. A single most-parsimonious tree (length $=910$, $\mathrm{CI}$ excluding uninformative characters $=0.56$, retention index $=0.72$ ) was obtained by setting the cytochrome $b$ sequence data under unweighted parsimony (Figure 3 ).

The tree generated from MP analysis revealed a similar topology (Figure 3) to that from the ML and NJ methods, but with a few differences. Only bootstrap values higher than $50 \%$ are displayed. Overall, the phylogenetic trees obtained using MP methods divide the species into five clades (Figure 3). Group I consists of G. flavimarginatus, G. chilospilus, G. reticularis, G. fimbriatus, G. javanicus, G. rueppeliae, G. curostus, G. reevesi, G. pseudothyrsoideus, G. kidako (from Japan), and G. polygonius (from Spain). Strong bootstrap values $(100 \%)$ exist for the formation of this clade. The internal branch of group I is also very clear. Interestingly, the Japanese species G. kidako is grouped with the Spanish species G. polygonius (bootstrap values $=65 \%$ ), and the other species of Gymnothorax within group 
I formed a cluster, supported by a high bootstrap value (100\%), in which G. javanicus, $G$. rueppeliae, and $G$. curostus are a separate branch (bootstrap values $=66 \%$ ). Group I then puts G. flavimarginatus, G. chilospilus, G. reticularis, and G. fimbriatus into a larger cluster (bootstrap value $=91 \%$ ). Furthermore, G. pseudothyrsoideus and G. reevesi are formed into two separate internal branches within group I (bootstrap values $=99 \%$ and $100 \%$ ).

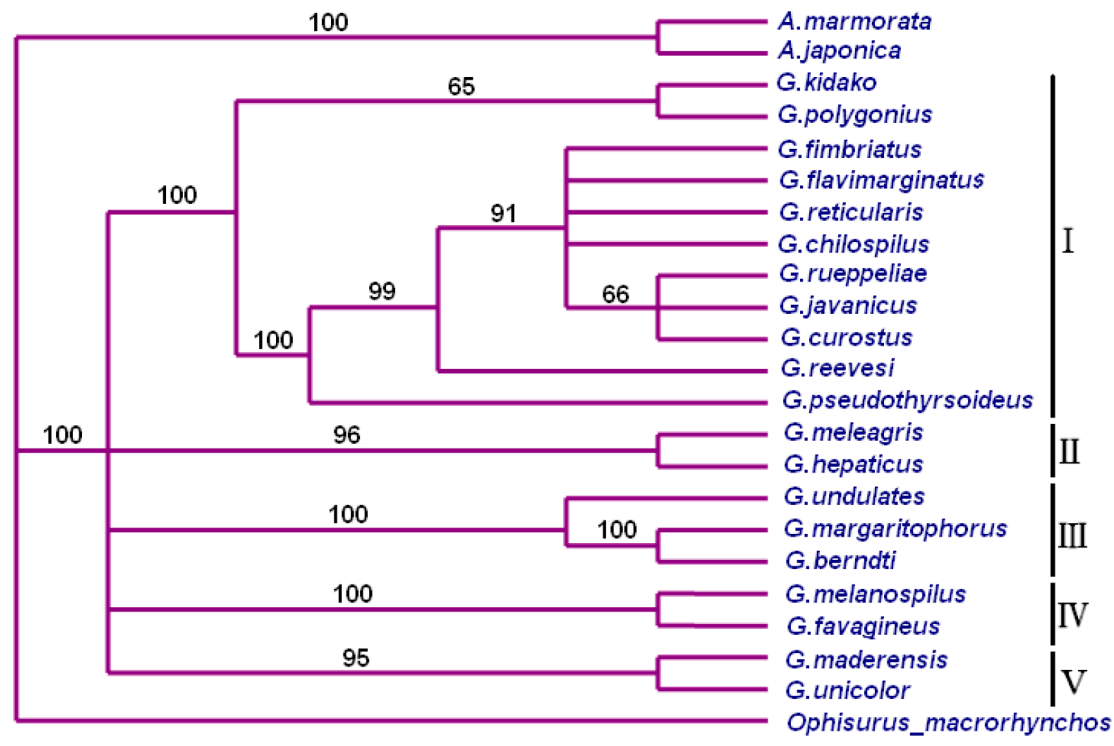

Figure 3. Most-parsimonious tree $(L=910$ steps, $C I=0.56, R I=0.72)$ resulting from the unweighted analysis of the cytochrome $b$ sequences. Numbers at the nodes represent bootstrap values (\%) with 1000 replicates. Only bootstrap values $>50 \%$ are shown.

Group II contains G. meleagris and G. hepatious (bootstrap value $=96 \%$ ). Group III is composed of G. margaritophorus, G. berndti, and G. undulates, which reveals a close genetic relationship between them (bootstrap value $=100 \%$ ). Group IV consists of $G$. melanospilus and G. favagineus (bootstrap value $=100 \%$ ), and Group V contains G. unicolor and G. maderensis, which originate from Spain (bootstrap value $=95 \%$ ).

The NJ analysis produced a tree topology identical to that of the ML analysis (-ln $\mathrm{L}$, unconstrained $=4707.71777)$. Thus, we combined the trees from both methods into a single tree represented by the NJ tree (Figure 4). The NJ tree divides the species into three clades. Group I consists of G. flavimarginatus, G. chilospilus, G. reticularis, G. fimbriatus, G. javanicus, G. rueppeliae, G. curostus, G. reevesi, G. pseudothyrsoideus, G. kidako, and G. polygonius, which is consistent with group I in the MP tree. Strong bootstrap values exist for the formation of this clade (bootstrap values $=100 \%$ ). For the other clades, however, are a few differences between the MP tree and the NJ tree. Group II contains G. margaritophorus, G. berndti, G. undulates, G. meleagris, G. hepatious, G. melanospilus, and G. favagineus for the NJ tree, which reveals the close genetic relationship between them. The internal branch of group II is also very clear. It consists of two subgroups, which are divided by an internal node. The subgroup IIA includes G. margaritophorus, G. berndti, G. undulates, G. meleagris, and G. hepatious; G. melanospilus and G. favagineus are gathered into subgroup IIB. However, 
the topology of these species for the MP tree is divided into three clades (group II, group III, and group IV). The two Spanish species (G. unicolor and G. maderensis) formed a separate evolutionary branch (group III), which is also consistent with the MP tree (group V).

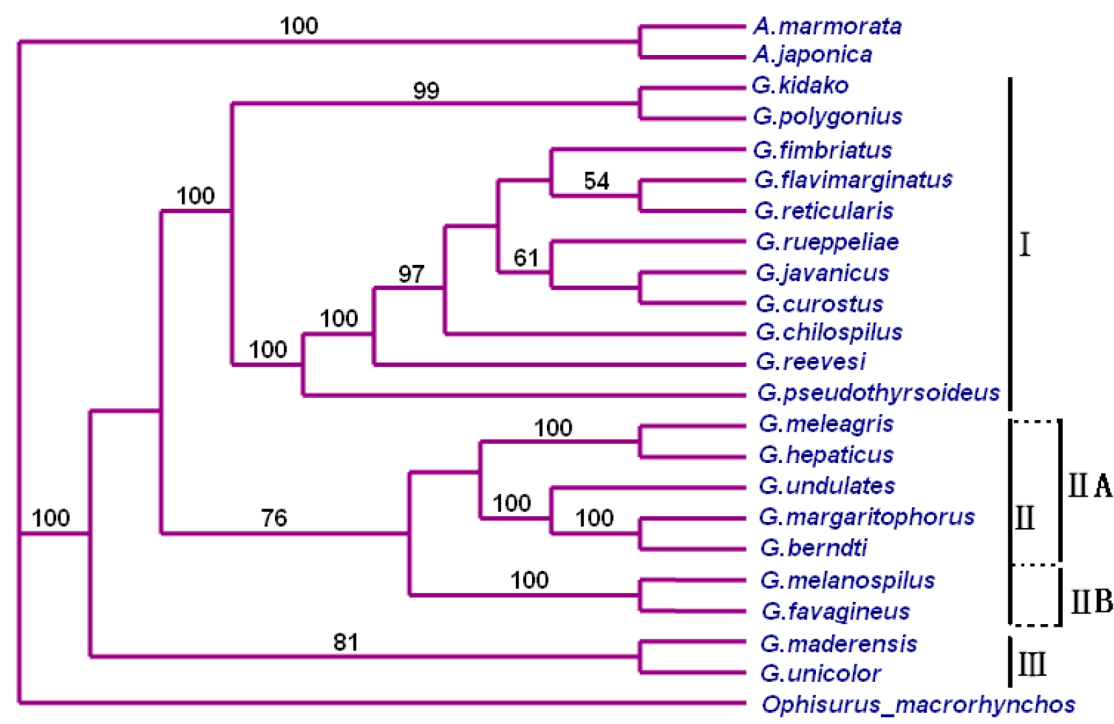

Figure 4. Neighbor-joining tree depicting relationships for 20 Gymnothorax species (moray eels) based upon gene sequences encoding cytochrome $b$. This tree resulted from the maximum likelihood analysis (PAUP* 4.0b10) following the GTR (general-time-reversible model; Goldman and Yang, 1994). Numbers indicate bootstrap values of 10 replicates. The ML tree (-in $L=4707.71777)$ was highly congruent and identified the same topology. Values at branches are NJ bootstrap estimates, based on 1000 replicates. Only bootstrap values $>50 \%$ are shown.

\section{DISCUSSION}

Moray eels were given the genus name Gymnothorax by Bloch in 1795 (Cheng and Zheng, 1987). Conventionally, coloration pattern, variegated markings, and dentition are used as the major morphological features for species identification (Cheng and Zheng, 1987). However, because a moray eel's color patterns/markings vary during different growth stages, and morphological features of different species often overlap, it is very difficult to apply these morphological identification methods accurately. For this reason, researchers have argued for many years about the richness of Gymnothorax species within the same geological area. For example, Cheng and Zheng (1987) believe there are 20 Gymnothorax species in the South and East China Seas. Shen (1993) however, maintains that there are 27 Gymnothorax species. In our study, in the South China Sea alone, we found 16 Gymnothorax species. There may be two reasons for this: 1) certain species have become extinct, or came close to extinction, due to the deterioration of the marine environment in the recent years; 2) Some species may only reside in the East China Sea, which we did not sample. Concerning the distribution of fish resources, G. undulates is the most abundant species of Gymnothorax in the South China Sea. Additionally, G. fimbriatus, G. flavimarginatus, G. meleagris, and G. favagineus are also the abundant species. 
In the present study, we constructed a phylogenetic tree based on 16 Gymnothorax species that inhabit the South China Sea, and combined this data with three species of Gymnothorax originating from Spain as well as a Japanese species. Our phylogenetic analysis indicates that the cytochrome $b$ data matrix contains a phylogenetic signal. Base substitution saturation analysis suggested that for both third codon positions, and the full-length cytochrome $b$ data set, Ts are not saturated, but Tv substitutions may be saturated.

Our phylogenetic tree showed that the genus Gymnothorax is divided into five distinct clades for the MP tree, and three clades for the NJ tree. The adult G. melanospilus and G. favagineus are morphologically similar, except that the shape and the spacing of spots on the body surface vary. Shih-chieh Shen argue that G. melanospilus and G. favagineus are the same species (Shen, 1993), and named it G. favagineus, but Cheng and Zheng had proposed that they are two different species (Cheng and Zheng, 1987); they were also divided into two species in FishBase. Based on our experimental data, the pairwise distance between $G$. melanospilus and G. favagineus is only 0.005, thus, we share Shih-chieh Shen's view in that these are the same species (Shen, 1993). To further resolve the issue, more genes should be sequenced, both mitochondrial and nuclear.

In this study, we conducted a preliminary investigation into the resource distribution of Gymnothorax (moray eels) in the South China Sea. We collected a total of 16 Gymnothorax species, as mentioned above. The phylogenetic relationships between those 16 Gymnothorax species were clarified according to the complete gene sequences encoding the mitochondrial protein cytochrome $b$.

\section{Conflicts of interest}

The authors declare no conflict of interest.

\section{ACKNOWLEDGMENTS}

We are grateful to all members of the Key Laboratory of Tropical Marine Biology Resources, College of Ocean in Hainan University, for their excellent technical assistance and encouragement. Research supported in part by the National Natural Science Foundation of China (\#30770283, \#31360638), the National Key Technology R\&D Program of China (\#2007BAD29B03), the Natural Science Foundation of Hainan Province (\#808122), the Opening Foundation of Key Laboratory of Tropical Marine Biology Resources (shkyjj0801), the young academic leaders of reserve talent project in Yunnan Province (\#2015HB059), the Science Research Fund of Yunnan Provincial Department of Education (\#ZD2013009), and the young academic leaders of reserve talent project in Honghe University (\#2014HB0203), Hong he University doctoral special project (\#14bs11).

\section{REFERENCES}

Avise JC (1994). Molecular Markers, Natural History and Evolution. New York, London: Champman \& Hall. 511.

Bohlke JE and Chaplin CCG (1993). Fishes of the Bahamas and adjacent tropical waters. 2nd edn. University of Texas Press, Austin.

Bernardi G, Findley L and Rocha-Olivares A (2003). Vicariance and dispersal across Baja California in disjunct marine fish populations. Evolution 57: 1599-1609. http://dx.doi.org/10.1111/j.0014-3820.2003.tb00367.x

Briolay J, Galtier N, Brito RM and Bouvet Y (1998). Molecular phylogeny of Cyprinidae inferred from cytochrome $b$ 
DNA sequences. Mol. Phylogenet. Evol. 9: 100-108. http://dx.doi.org/10.1006/mpev.1997.0441

Cantatore P, Roberti M, Pesole G, Ludovico A, et al. (1994). Evolutionary analysis of cytochrome $b$ sequences in some Perciformes: evidence for a slower rate of evolution than in mammals. J. Mol. Evol. 39: 589-597. http://dx.doi. org/10.1007/BF00160404

Castle PHJ in Gomon MF, Glover JCM and Kuiter RH (Eds) (1994). The Fishes of Australia's South Coast. State Print, Adelaide, 992.

Cheng QT and Zheng BS (1987). Systematic Synopsis of Chinese Fishes. Beijing: Science Press, 750-755.

Felsenstein J (1985). Confidence limits on phylogenies: an approach using the bootstrap. Evolution 39: 783-791. http:// dx.doi.org $/ 10.2307 / 2408678$

Gilbert M, Rasmussen JB and Kramer DL (2005). Estimating the density and biomass of moray eels (Muraenidae) using a modified visual census method for hole-dwelling reef fauna. Environ. Biol. Fishes 73: 415-426. http://dx.doi. org/10.1007/s10641-005-2228-2

Goldman N and Yang Z (1994). A codon-based model of nucleotide substitution for protein-coding DNA sequences. Mol. Biol. Evol. 11: 725-736.

Harris PM, Mayden RL, Espinosa HS and de Leon FG (2002). Phylogenetic relationships of Moxostoma and Scartomyzon (Catostomidae) based on mitochondrial cytochrome $b$ sequence data. J. Fish Biol. 61: 1433-1452. http://dx.doi. org/10.1111/j.1095-8649.2002.tb02488.x

Hillis DM and Huelsenbeck JP (1992). Signal, noise, and reliability in molecular phylogenetic analyses. J. Hered. 83: 189-195.

Inoue JG, Miya M, Tsukamoto K and Nishida M (2003). Basal actinopterygian relationships: a mitogenomic perspective on the phylogeny of the "ancient fish". Mol. Phylogenet. Evol. 26: 110-120. http://dx.doi.org/10.1016/S10557903(02)00331-7

Kimura M (1980). A simple method for estimating evolutionary rates of base substitutions through comparative studies of nucleotide sequences. J. Mol. Evol. 16: 111-120. http://dx.doi.org/10.1007/BF01731581

Kocher TD, Thomas WK, Meyer A, Edwards SV, et al. (1989). Dynamics of mitochondrial DNA evolution in animals: amplification and sequencing with conserved primers. Proc. Natl. Acad. Sci. USA 86: 6196-6200. http://dx.doi. org/10.1073/pnas.86.16.6196

Kuiter RH. (1996). Guide to Sea Fishes of Australia. New Holland: 433.

Ling YJ, Iizuka Y and Tzeng WN (2005). Decreased Sr/Ca ratios in the otoliths of two marine eels, Gymnothorax reticularis and Muraenesox cinereus, during metamorphosis. Mar. Ecol. 304: 201-206. http://dx.doi.org/10.3354/ meps304201

Lowe-McConnell RH (1987). Ecological studies in tropical fish communities. Cambridge University Press, London.

Martin AP (1995). Mitochondrial DNA sequence evolution in sharks: rates, patterns, and phylogenetic inferences. Mol. Biol. Evol. 12: 1114-1123.

Martin AP and Bermingham E (1998). Systematics and evolution of lower Central American cichlids inferred from analysis of cytochrome $b$ gene sequences. Mol. Phylogenet. Evol. 9: 192-203. http://dx.doi.org/10.1006/mpev.1997.0461

Paxton JR and Eschmeyer WN (1994). Encyclopedia of Fishes. Sydney, New South Wales University Press, San Diego, Academic Press, 240.

Perdices A, Cunha C and Coelho MM (2004). Phylogenetic structure of Zacco platypus (Teleostei, Cyprinidae) populations on the upper and middle Chang Jiang (=Yangtze) drainage inferred from cytochrome $b$ sequences. Mol. Phylogenet. Evol. 31: 192-203. http://dx.doi.org/10.1016/j.ympev.2003.07.001

Randall JE (1996). Caribbean Reef Fishes. 3rd edn. T.F.H. Publications, Neptune.

Rogers JS and Swofford DL (1999). Multiple local maxima for likelihoods of phylogenetic trees: a simulation study. Mol. Biol. Evol. 16: 1079-1085. http://dx.doi.org/10.1093/oxfordjournals.molbev.a026197

Shen SC (1993). Fishes of Taiwan. Taibei, Department of Zoology, National Taiwan University, 101-107.

Song CB, Near TJ and Page LM (1998). Phylogenetic relations among percid fishes as inferred from mitochondrial cytochrome $b$ DNA sequence data. Mol. Phylogenet. Evol. 10: 343-353. http://dx.doi.org/10.1006/mpev.1998.0542

Stepien CA, Kocher TD (1997). Molecules and morphology in studies of fish evolution. Mol Sys Fishes: 1-11.

Swofford DL (2002). PAUP*: Phylogenetic Analysis Using Parsimony (*and Other Methods), Version 4.0 beta 10. Sinauer Associates, Sunderland, MA.

Tamburrini M, Verde C, Olianas A, Giardina B, et al. (2001). The hemoglobin system of the brown moray Gymnothorax unicolor: structure/function relationships. Eur. J. Biochem. 268: 4104-4111. http://dx.doi.org/10.1046/j.14321327.2001.02333.X

Tamura K, Peterson D, Peterson N, Stecher G, et al. (2011). MEGA5: molecular evolutionary genetics analysis using maximum likelihood, evolutionary distance, and maximum parsimony methods. Mol. Biol. Evol. 28: 2731-2739. 
http://dx.doi.org/10.1093/molbev/msr121

Tesch FW (1977). The Eel: Biology and Management of Anguillid Eels. Chapman \& Hall. 434.

Thomas JN, Porterfield JC and Page LM (2000). Evolution of Cytochrome $b$ and the Molecular Systematics of Ammocrypta (Percidae: Etheostomatinae). Copeia 3: 701-711.

Wu XD, Xiao W, Lian ZQ and Hou YX (2014). The Cloning and Sequence Analysis of the Mitochondrial Cytochrome $b$ Gene of Silurus Lanzhouensis. Acta Hydrobiol. Sin. 38: 772-779.

Xiang F, Zou JX, Deng FJ, Liu SY, et al. (2004). The molecular taxonomy and phylogeny of Zebrafish (Danio rerio) based on the Cyt $b$ partial Sequences. Chin. J. Zool. 39: 13-18. 